\title{
Collision Warning Design to Mitigate Driver Distraction
}

\author{
John D. Lee \& Joshua D. Hoffman \\ Department of Mechanical and \\ Industrial Engineering, University of Iowa \\ Iowa City, IA, USA \\ jdlee@engineering.uiowa.edu \\ jhoffman@engineering.uiowa.edu
}

\author{
Elizabeth Hayes \\ Human Machine Interaction-USA \\ DaimlerChrysler Research and Technology \\ Portland, OR, USA \\ ElizabethHayes@Freightliner.com
}

\begin{abstract}
As computers and other information technology move into cars and trucks, distraction-related crashes are likely to become an important problem. This paper begins to address this problem by examining how alert strategy (graded and singlestage) and alert modality (haptic and auditory) affect how well collision warning systems mitigate distraction and direct drivers attention to the car ahead when it unexpectedly brakes. We conducted two experiments in which drivers interacted with an in-vehicle email system and a collision warning system signaled a braking lead vehicle. The first experiment showed that graded alerts led to a greater safety margin and a lower rate of inappropriate responses to nuisance warnings. A second experiment focused on attitudes toward the collision warning system and found that graded alerts were more trusted than single stage alerts and that haptic alerts, a vibrating seat in these experiments, were perceived as less annoying and more appropriate. Graded haptic alerts offer a promising approach to developing context aware computing in a safetycritical application.
\end{abstract}

\section{Categories \& Subject Descriptors: H 5.0 [General in- formation interface]}

General Terms: Design, Experimentation, Human Factors, Performance.

Keywords: Smart cars, Distraction, Collision warning systems, User acceptance, Trust, Notification systems.

\section{INTRODUCTION}

Just as computers have transformed the office in the last 20 years, they will likely transform the car in the next decade. Recent advances in sensor, wireless, computing, and Global Position System (GPS) technology make sophisticated invehicle information systems (IVIS) feasible. These advances, combined with societal trends for increased productivity and the diffusion of work beyond the traditional office environment, make these systems likely. Computer, software, tele-

\footnotetext{
Permission to make digital or hard copies of all or part of this work for personal or classroom use is granted without fee provided that copies are not made or distributed for profit or commercial advantage and that copies bear this notice and the full citation on the first page. To copy otherwise, or republish, to post on servers or to redistribute to lists, requires prior specific permission and/or a fee.

CHI 2004, April 24-29, 2004, Vienna, Austria.

Copyright 2004 ACM 1-58113-702-8/04/0004 ...\$5.00.
}

communications, and automotive companies have begun to develop IVIS functions in anticipation of a $\$ 15-\$ 100$ billion market [1, 15, 16]. Representative IVIS functions include voice-based email access, scrolling text messages, and turnby-turn navigation aids.

Because these functions must be shared with driving, notification systems are a critical and relatively unexplored research issue that may govern the success of IVIS [19]. Unlike the desktop domain, IVIS functions require timesharing with the safety-critical task of driving. Many studies have demonstrated the distraction potential of cellular telephones $[4,10]$ and recent studies have also shown that voice-based email systems [13] and navigation systems can also distract drivers [22].

Drivers who fail to timeshare IVIS interactions and neglect potential collision situations could benefit from a notification system that provides warnings that alert them to an impending collision. These warnings include those that alert the driver to a braking vehicle ahead (rear-end collision warning) and those that alert the driver as he or she drifts towards the edge of the road (road departure warnings). Designing warnings that promote appropriate responses and acceptance is a ubiquitous problem that confronts designers of many systems. An inappropriate response occurs when the driver responds to the situation incorrectly, such as ignoring the collision warning and failing to brake when necessary, or braking sharply when it was not necessary to brake. Either of these inappropriate responses could jeopardize a driver's safety.

The success of collision warning systems depends on how well the algorithm and driver interface are tailored to driver capabilities and preferences. A series of recent studies have investigated how various algorithms $[5,26]$ and algorithm parameters $[6,15,21]$ influence collision warning effectiveness. Algorithms calculate when to issue a warning and have a strong effect on the safety benefit of collision warning systems, but the driver interface may have an equally important effect. The driver interface influences how quickly the driver responds and whether the driver will accept the system. A loud auditory warning might generate a quick response, but if it occurs frequently it could rapidly undermine driver acceptance.

Two critical factors govern the effectiveness of collision warning systems. First, the collision warning system must promote a timely and appropriate driver response. Second, 
annoyance associated with nuisance warnings must be minimized and drivers must trust the system if drivers are to accept it $[2,12]$. This paper describes two experiments that investigate how driver interface alternatives affect driver performance and attitude.

Graded and single-stage warnings represent distinct strategies of presenting warning information that have important implications for driver performance and acceptance. A graded warning presents a signal proportional to the degree of threat, such as a louder auditory signal as the driver approaches a lead vehicle. A single-stage warning provides signal only when the degree of threat exceeds a threshold. A graded warning might enhance driver response by priming the drivers' response and enhance their understanding and trust in the system [17]. A graded warning is an example of a likelihood alarm display (LAD), where information about event likelihood is computed by an automated monitoring system and encoded into a warning signal. Sorkin, Kantowitz, and Kantowitz [25] evaluated operator performance within a dual-task paradigm with two LADS: a color-coded visual alarm and a linguistically coded synthetic speech alarm. The results indicated that LADs can improve the allocation of attention among tasks; however, the additional information provided by LADs might add to the drivers' attentional load. Thus, the benefits of a graded warning may be outweighed if their frequent occurrence annoys or distracts drivers.

Another important interface characteristic that could affect driver performance and acceptance is the sensory modality in which the warning is presented. Haptic cues offer a promising and relatively unexplored alternative to auditory warnings that could speed response and reduce annoyance. In particular, torque-based kinesthetic cues reduced reaction times more than auditory cues [8] and vibrotactile cues enhanced reaction time to visual cues [7]. Substantial research shows that complementing visual cues with redundant cues in another sensory mode speeds reaction time [20, 28]. Haptic warnings have also proved more effective in alerting pilots to mode changes in cockpit automation compared to visual cues. Pilots receiving visual alerts detected $83 \%$ of the mode changes; those with haptic alerts detected $100 \%$ of the mode changes. In addition, the haptic cues did not interfere with performance of concurrent visual tasks [24]. These findings show that haptic feedback is a promising method of alerting people in event-driven, information-rich domains.

In the driving domain, several studies have found haptic displays improve driver reaction to collision situations. Janssen and Nilsson [11] compared headway adjustments of drivers who were alerted with a light, a warning buzzer, and a 'smart' gas pedal, which produced a $25 \mathrm{~N}$ increase in pedal force whenever the driver got dangerously close to the vehicle ahead. The 'smart' gas pedal generated the greatest safety benefit. Similarly, Tijerina et al. [27] investigated haptic cues for rear-end collision warnings and found promising results for a mono-pulse braking cue. While seat vibration has been used as a lane departure warning [23], it has not been used as a longitudinal alert. Unlike the gas pedal feedback and the brake pulse, seat vibration does not have a natural mapping to a braking response and may perform more poorly than the more commonly used visual and auditory display modalities. Understanding the relative merits of auditory and seat-based vibration warnings has important implications for mitigating driver distraction with a collision warning system.

This paper describes two experiments. Experiment 1 has two primary objectives: 1) examine how driver response depends on graded and single-stage warning strategies; 2) examine how driver response depends on the modality of the warning display, such as seat vibration. The primary objective of Experiment 2 is to examine how these warning strategies and modalities affect driver preferences.

\section{EXPERIMENT 1: DRIVER PERFORMANCE}

\section{Method}

A mixed between/within-subject experimental design contrasted graded and single-stage warning strategies with auditory and haptic warning modalities. Participants drove three 15-minute scenarios in a fixed-base driving simulator and experienced 21 braking events that were comprised of three levels of severity. Two thirds of these events did not require any driver response, but could trigger a warning. Because only severe braking events trigger the single-stage warnings drivers in the single-stage warning collision condition received only three warnings, whereas those with the graded warning strategy received 21 warnings. All participants used an interactive speech-based email system during all scenarios.

\section{Participants}

Forty individuals, 20 females and 20 males between the ages of 25 and 55 (mean 37.2, standard deviation 9.1) participated. All drivers were licensed and had normal or corrected normal vision. Drivers were unaware of the nature of the research prior to participation. All were compensated \$20 for their participation.

\section{Apparatus}

A fixed-based, medium-fidelity driving simulator was used in the experiment. The driving simulator uses a 1992 Mercury Sable configured with the Hyperion Technologies Vection Research Simulator. The simulator is a fully integrated, highperformance driving simulation system designed for use in ground vehicle research and training applications. The simulator uses a real vehicle cab (Mercury Sable) that includes a 50-degree visual field of view, full instrumentation with actual gauges, force feedback operator controls, and a rich audio environment. The fully textured graphics are generated by PC hardware that delivers a $60 \mathrm{~Hz}$ frame rate at $1024 \times 768$ resolution.

The simulator was equipped with a color $640 \times 480$ pixel screen mounted on the dash of the car in a high head-down position. A visual collision warning icon was presented on this screen, approximately one meter in front of the driver just 
above the instrument cluster ( 7 degrees below the drivers' eye point). The car was also equipped so that the collision warning sound could be played on a MIDI device through standard PC speakers. The front seat was also modified to include actuators on the front edge and in the thigh bolsters of the seat that vibrated to generate the haptic cues. The loudness of the auditory warnings was recorded in the vehicle while the simulator was running: severe level $74.3 \mathrm{~dB}$, moderate level $62.5 \mathrm{~dB}$ and negligible level $53.7 \mathrm{~dB}$. The seat vibration mimicked the auditory warning and as the warning level became more severe, the intensity and frequency of the vibration increased. The auditory, visual or haptic warning signal persisted for as long as the driver was in a situation that was sufficient to trigger a warning.

\section{Experimental design and independent variables}

A mixed between-within subject design was used. The two between subject variables had two levels; warning modality (haptic and visual or auditory and visual) and warning strategy (graded or single-stage). There were 10 participants in each of these conditions. The two within subject variables were the severity of lead vehicle braking and whether a response was required. The severity of vehicle braking had three levels: severe, moderate, and negligible. The singlestage warning was triggered only by the severe braking events and the graded warning was triggered by all levels. It was not necessary for the driver to respond to all braking events despite the warning being triggered. Nuisance alarms occurred when the lead vehicle braked briefly and then accelerated. In these situations the driver could maintain a nearly constant headway without braking. With the single-stage warning drivers received one nuisance alert in each of the first two drives, and a true warning on the final drive. In the graded alert conditions drivers received an alert for each braking event for a total of seven per drive.

\section{Dependent variables}

Several dependent variables were used to characterize the safety benefit and driver response process. There two variables were used to estimate the safety benefit of the collision warning - the number of collisions and adjusted minimum time to collision. Adjusted minimum time to collision is a continuous measure of safety margin, and is calculated using equations of motion to determine how long it will take the two vehicles to collide at their current relative position, velocity, and acceleration [14]. If the drivers collide, the adjusted minimum time to collision is calculated as the time at which the driver would have needed to begin braking to avoid the collision, given the driver's deceleration at the point of collision. The drivers' response process was described according to three reaction time variables: onset of lead vehicle braking to the accelerator release, movement time from accelerator release to initial brake press, and initial brake press to maximum deceleration. Drivers' response was also described by the mean and maximum deceleration. Mean deceleration was defined as the mean deceleration of the vehicle from the onset of braking until the driver's vehicle stopped, collided with the lead vehicle, or the driver began to accelerate after the braking event. Maximum deceleration was defined as the peak deceleration between the start and end of the braking event. These measures are important because they help to define inappropriate responses. An inappropriate response occurred when the driver responded to the situation incorrectly, such as ignoring the collision alarm and failing to brake when necessary, or braking sharply when it was not necessary to brake.

\section{Procedure}

Upon arrival, participants completed an informed consent form and were instructed about how to operate the simulator and interact with an auditory email system. The experiment consisted of an introductory drive (5 minutes) and three longer (15 minute) drives that contained braking events interspersed with routine highway driving. Participants were instructed to follow the lead vehicle at a speed of $88.5 \mathrm{kh}(55$ $\mathrm{mph}$ ). The introductory drive consisted of a single braking event, which the driver was told about beforehand. The lead vehicle braked seven times during each of the three longer drives. The braking events were organized in a stratified random distribution, with each scenario containing an equal number of each event severity. Only one severe braking event required a response and it occurred at the end of the last drive.

During the drive, participants were asked to complete an auditory email task. The purpose of this task was to provide a degree of distraction similar to what may be present when a collision warning system might generate a warning in an actual on-road situation. Previous research has shown this system to generate a $30 \%$ increase in drivers brake response [13]. The drivers' task was to listen to email menu options, select the appropriate option, listen to the content of the email, and then delete the message according to a simple criterion. The email messages pertained to four projects named after the four seasons; winter, spring, summer, and fall. The driver was instructed to listen to the emails and delete any message that pertained to the "fall" project. The other messages were to be left unchanged. The email system began automatically, and the drivers controlled the email system by speaking a menu option when the system had finished giving the choices. For example, to delete a message, drivers would say, "Delete message" after the options had been spoken by the email system. The total length of participation was approximately 70 minutes.

\section{Results and Discussion}

Data from 40 subjects were compiled to form a database of 840 braking events. Because several drivers failed to maintain the correct speed, the lead vehicle was too far ahead when braking events occurred, and so the braking event was considered invalid. Removing the invalid events from the final dataset resulted in a total of 741 data points. A repeatedmeasures ANOVA was used to analyze the data using twotailed hypothesis tests. 


\section{Severity of braking events and driver response}

Drivers generally responded to braking events in a systematic and realistic manner. For example, the adjusted minimum time to collision reflected the braking severity of the lead vehicle, with the negligible event having the largest adjusted minimum TTC $(6.04 \mathrm{~s})$, followed by the moderate $(4.97 \mathrm{~s})$ and then by the severe event $(2.03 \mathrm{~s}), F(2,72)=172.08, p<0.0001$. The severity of the lead vehicle braking also affected drivers' braking response, with the most severe braking events having the greatest maximum deceleration $\left(3.36 \mathrm{~m} / \mathrm{s}^{2}\right)$, followed by moderate events $\left(1.54 \mathrm{~m} / \mathrm{s}^{2}\right)$ and negligible events $\left(1.06 \mathrm{~m} / \mathrm{s}^{2}\right)$, $F(2,72)=384.85, p<0.0001$. Likewise, severe braking events had the greatest mean deceleration $\left(1.76 \mathrm{~m} / \mathrm{s}^{2}\right)$, followed by moderate events $\left(0.690 \mathrm{~m} / \mathrm{s}^{2}\right)$ and negligible events $(0.439$ $\left.\mathrm{m} / \mathrm{s}^{2}\right), F(2,72)=363.20, p<0.0001$. The true warning and nuisance alarm conditions also had the expected effects, with nuisance alarms (5.52s) having a larger adjusted minimum TTC than true warnings (4.46s), $F(1,36)=94.03, p<0.0001$. This response was also reflected in the maximum deceleration, with true warnings having a much greater deceleration $\left(3.12 \mathrm{~m} / \mathrm{s}^{2}\right)$ compared with the nuisance alarms $\left(0.66 \mathrm{~m} / \mathrm{s}^{2}\right)$, $F(1,36)=1458.26, p<0.0001$. The mean acceleration showed a similar pattern, with drivers braking harder for the true warnings $\left(1.61 \mathrm{~m} / \mathrm{s}^{2}\right)$ compared to the nuisance alarm events $\left(0.201 \mathrm{~m} / \mathrm{s}^{2}\right), F(1,36)=1380.76, p<0.0001$. Overall, manipulating the braking event severity affected the demands of the driving situation and drivers responded realistically: deceleration levels were similar to those seen in other studies and drivers distinguished between situations that required them to brake and those that did not. These results suggest that drivers quickly adapted to the driving simulator and responded in a manner similar to actual driving situations.

\section{Interface characteristics and safety benefit}

The headway and the braking behavior of the lead vehicle placed the participants' vehicle in a collision situation only when the braking event was severe, not when the event was moderate or negligible. This situation occurred once for each participant at the end of the experiment. Therefore, the design of the experiment allowed for a maximum of 40 collisions to occur. Ten collisions occurred in the study. Seven occurred in the single-stage warning condition and three in the graded warning condition, $X^{2}(1)=2.13, p=0.144$. The collisions were evenly divided between the interface modalities, with five collisions occurring in the auditory and five occurring in the haptic condition. Of the collisions in the graded condition, two occurred with the haptic warning and one with the auditory warning. For this relatively short, simulated drive, these results suggest a potential benefit of the graded warning and show no indication that a graded warning might habituate drivers to warnings and impair their response to single-stage collision situations. However, crashes are infrequent and an insensitive measure of the safety margin produced by the collision warning system.

A more sensitive measure is the adjusted minimum time to collision, which shows a slight benefit for the graded warning (5.18s) compared to the single-stage warning (5.15s), $F(1,36)=8.74, p=0.0055$. Figure 1 shows how this main effect is complicated by interactions with the braking event severity, $F(1,72)=14.87, \quad p<0.0001$, the accuracy of the alert, $F(1,36)=23.56, p<0.0001$, and a three way interaction between the braking event severity, accuracy of the alert, and the alert strategy, $F(1,71)=13.86, p<0.0001$. For true warnings, the performance was similar with the negligible and moderate collision situations, but the graded warning provides a substantial safety benefit for the severe braking event. This suggests that drivers are capable of detecting possible collision situations of lower severity which coincide with the lower levels of the graded warning. Therefore, the lower levels of the graded warning may either be complementary to drivers' natural response, or unnecessary, but the graded warning provides a substantial benefit in severe braking situations by giving the driver an early indication of an evolving collision situation, something the single-stage warning does not.
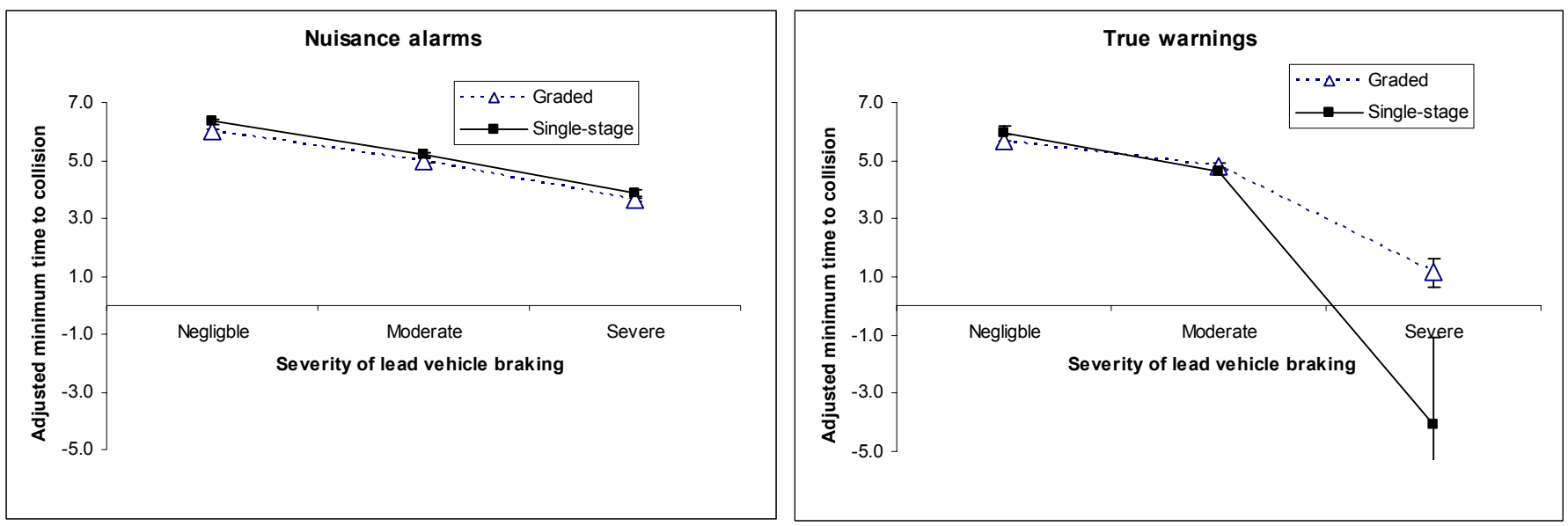

Figure 1. The effect of braking event severity, warning accuracy, and warning strategy on adjusted minimum TTC. 


\section{Interface characteristics and response process}

The braking response process is composed of three components: the time between the onset of the lead vehicle braking to the accelerator release, accelerator release to initial brake press, and initial brake press to maximum brake application. Following the release of the accelerator the car begins to decelerate and when the brakes are pressed deceleration increases. The mean and maximum decelerations describe the degree of the braking response.

No significant effects were found for warning modality, but the maximum and mean deceleration both depend on an interaction between the severity of the braking event and the warning strategy. Graded warnings induced greater maximum deceleration in the moderate braking condition, $F(2,72)=9.00$, $p=0.0003$. This effect is replicated in the mean deceleration data, $F(2,72)=6.53, p=0.0025$. This result suggests that the graded warning prompts cautionary braking that can preserve driver safety if the lead vehicle begins to brake more severely.

The nuisance alarms prompted a significantly faster accelerator release $(0.665 \mathrm{~s})$ than the true warnings $(1.230 \mathrm{~s})$, $F(1,36)=60.7, p<0.0001$, but only for negligible and moderate braking events, $F(2,64)=17.02, p<0.0001$. This somewhat counterintuitive result reflects the fast response of a relatively few drivers who responded to the nuisance alarms. Those that responded to the nuisance alarms seem to respond by quickly removing their foot from the accelerator and then assessing the situation. For other drivers, the warning directs their attention to the road and then they respond if the situation warrants it. The response-followed-by-assessment strategy generates a response to nuisance alarms and a faster reaction time compared to the assessment-followed-by-response strategy. A previous study of collision warnings showed a similar pattern of response strategies in which some drivers respond immediately to the warning and others use the warning as a cue to assess the situation [15]. The experimental manipulations did not affect accelerator to brake and the brake to maximum brake transition times.

\section{Interface characteristics and response appropriateness}

The aggregate mean and maximum deceleration data show little evidence that drivers respond inappropriately to the collision warnings. There was little tendency for drivers to brake when the situation did not demand it. The validation of the experimental conditions shows that drivers' braking was generally attuned to the severity of the situation. Although the aggregate data suggests that the warnings did not induce inappropriate behavior, it is possible that the aggregate data fail to represent the behavior of individual drivers. For this reason, the appropriateness of driver response to the warnings was examined by assigning the values of the maximum and mean deceleration for each braking event to levels 0 through 4. Deceleration values of zero $g$ or below were assigned a response level of 0 , indicating situations with no deceleration or slight acceleration. Deceleration values of $0 \mathrm{~g}$ to $0.1 \mathrm{~g}$ $\left(0.98 \mathrm{~m} / \mathrm{s}^{2}\right)$ were assigned to level 1 . These values correspond to a deceleration achieved by releasing the foot from the accelerator, but not applying the brake. Deceleration values of $0.1 \mathrm{~g}$ to $0.25 \mathrm{~g}\left(2.45 \mathrm{~m} / \mathrm{s}^{2}\right)$ were assigned to level 2 , a mild cautious braking response. Deceleration values of 0.25 to 0.5 $\mathrm{g}\left(4.9 \mathrm{~m} / \mathrm{s}^{2}\right)$ were assigned to level 3 , indicating a moderate braking. Deceleration values of $0.5 \mathrm{~g}$ and greater were assigned to level 4, which denotes severe braking. Response levels of 3 and 4 indicate appropriate responses to the true warnings, while response levels of 0 and 1 indicate appropriate responses for nuisance alarms. Table 1 shows the frequency of responses for the nuisance alarm braking events. The $X^{2}$ analysis of these data shows that only the warning strategy affects the type of response, $X^{2}(1)=16.07, p=0.0011$. There were no severe braking reactions in response to either warning strategy, but there was a tendency for the single-stage warning to induce inappropriate cautionary and moderate braking. In contrast, the graded warning induced a large number of accelerator releases, which is an appropriate response that may enhance safety in those cases where the mild braking of the lead vehicle evolves into severe braking.

Table 1. Response types associated with the various experimental conditions for the nuisance alarm braking events.

\begin{tabular}{|c|c|c|c|c|c|c|c|}
\hline & & $\begin{array}{r}1 \mathrm{Nc} \\
\mathrm{I}\end{array}$ & $\begin{array}{l}0 \\
\text { Deceleration }\end{array}$ & $\begin{array}{c}2 \text { Accelerator } \\
\text { Release }\end{array}$ & $\begin{array}{l}3 \text { Cautious } \\
\text { ing }\end{array}$ & $\begin{array}{r}\text { Brak-4 Moderate } \\
\text { Braking }\end{array}$ & $\begin{array}{r}5 \text { Severe } \\
\text { Braking }\end{array}$ \\
\hline $\begin{array}{l}\text { Frequency } \\
(\% \text { of total })\end{array}$ & & 140 & $(25 \%)$ & $395(71 \%)$ & $19(3.4 \%)$ & $2(0.4 \%)$ & 0 \\
\hline & Negligible & & $(64 \%)$ & $219(55 \%)$ & $8 \quad(42 \%)$ & 1 & 0 \\
\hline Event Type & Moderate & & $(31 \%)$ & $114(29 \%)$ & $1 \quad(5 \%)$ & 0 & 0 \\
\hline & Severe & 7 & $(5 \%)$ & $62 \quad(16 \%)$ & $10(53 \%)$ & 1 & 0 \\
\hline Modality & Haptic & & $(49 \%)$ & $206(52 \%)$ & $5 \quad(26 \%)$ & 1 & 0 \\
\hline & Auditory & & $(51 \%)$ & $189(48 \%)$ & $14(74 \%)$ & 1 & 0 \\
\hline Strategy & Graded & 24 & $(17 \%)$ & $245(62 \%)$ & $7 \quad(37 \%)$ & 0 & 0 \\
\hline Strategy & Single-stage & 116 & $(83 \%)$ & $150 \quad(38 \%)$ & $12(63 \%)$ & 2 & 0 \\
\hline
\end{tabular}




\section{EXPERIMENT 2: DRIVER ATTITUDES}

\section{Method}

A within subject experimental design contrasted graded and single-stage warning strategies with auditory and haptic warning modalities. Twenty participants drove four 10-minute scenarios in a fixed-base driving simulator and experienced 24 braking events that were comprised of three levels of severity. Two thirds of these events produced nuisance alarms that did not require any driver response, depending on the warning strategy. With the single-stage warning strategy only severe events triggered a warning, but with the graded strategy all events triggered a warning.

\section{Participants}

Twenty individuals, 11 females and 9 males between the ages of 25 and 55 (mean 30.6, standard deviation 5.9) participated. All drivers were licensed and had normal or corrected normal vision. Drivers were unaware of the nature of the research prior to participation. All were compensated \$20 for their participation.

\section{Apparatus}

The hardware and software described in Experiment 1 was also used in Experiment 2. Sound levels were also the same, as were LCD screen placement and its characteristics. This was done to allow comparisons between the experiments.

\section{Independent variables and experimental design}

A within subject design was used, examining the same variables as in Experiment 1. Warning modality again had two levels (haptic and visual or auditory and visual), as did warning strategy (graded or single-stage). The severity of lead vehicle braking and whether a response was required (nuisance alarm or true warning) was also the same as in Experiment 1 . The order of the modality and strategy conditions was counterbalanced using a latin square.

\section{Dependent variables}

Driver attitudes were measured using a series of subjective rating scales after each 10-minute drive. Drivers rated annoyance, urgency, appropriateness, and trust in the collision avoidance system. Drivers also rated their self-confidence in detecting potential collision situations. Upon completion of all trials they then comparatively ranked the warning systems regarding criteria that included perceived benefit, annoyance, urgency, and trust of the warnings.

\section{Procedure}

Upon arrival, participants completed an informed consent form and were instructed about how to operate the simulator. The experiment included an introductory drive (5 minutes) and four longer (10 minute) drives that contained several braking events interspersed with routine highway driving. Participants were instructed to follow the lead vehicle at a speed of $88.5 \mathrm{kh}(55 \mathrm{mph})$. The introductory drive consisted of a single braking event, which was described to the driver. The lead vehicle braked 6 times during each of the four longer drives. As in the braking events were organized in a stratified random distribution, with each scenario containing an equal number of each event severity. Following each drive, participants completed subjective ratings. The total length of participation was approximately one hour.

\section{Results and Discussion}

The results focus on the drivers comparative rankings. The 20 participants were asked to rank the warning modalities in order from 1 to 4 based on preference, with 1 being the least preferred and 4 being the most preferred. These nonparametric data violate the assumptions of a repeatedmeasures ANOVA and so we apply a Friedman's nonparametric analysis to determine significant differences between the rankings by means of a quasi-F value. Only when the Friedman's test showed a significant difference between conditions was a post-hoc multiple comparison performed using Fisher's least significant difference method.

Table 2 shows that drivers perceived the graded haptic warning to be a greater benefit than a graded auditory or singlestage auditory warning, $F(3,45)=12.07, p<0.05$. Drivers showed that they would be more likely to buy a graded or single-stage haptic warning system than an single-stage auditory system, $F(3,45)=12.79, p<0.05$, and would be more likely to buy a haptic warning than an auditory warning, $F(3,45)=5.31, p<0.05$. Drivers trusted the graded haptic warning more than the graded auditory or single-stage auditory warnings, $F(3,45)=7.00, p<0.05$. The single-stage auditory warning was ranked as more annoying than the singlestage haptic and graded haptic warnings, $F(3,45)=15.95$, $p<0.05$.

The significance tests show an overall difference for urgency and overall preference across conditions, but the post-hoc tests fail to reach statistical significance. However, the trends suggests that single-stage auditory warnings are slightly more urgency and that the graded haptic alerts are viewed as most appropriate. 
Table 2. Summary of warning rank analysis, with higher values indicating higher rank.

\begin{tabular}{|c|c|c|c|c|c|c|}
\hline \multirow[b]{2}{*}{ Assessment } & & \multicolumn{4}{|c|}{ Warning Interface Characteristics } & \multirow[b]{2}{*}{$\begin{array}{l}\mathrm{F}(3,45) \\
\mathrm{p}<0.05\end{array}$} \\
\hline & & $\begin{array}{l}\text { Graded } \\
\text { Haptic }\end{array}$ & $\begin{array}{l}\text { Graded } \\
\text { Auditory }\end{array}$ & $\begin{array}{l}\text { Single-stage } \\
\text { Haptic }\end{array}$ & $\begin{array}{l}\text { Single-stage } \\
\text { Auditory }\end{array}$ & \\
\hline \multirow{4}{*}{ Benefit to Driving } & & $\Gamma$ & 7 & & & \multirow{3}{*}{12.07} \\
\hline & Mean & 3.06 & 2.13 & 2.88 & 2.06 & \\
\hline & Median & 3 & 2 & 3 & 2 & \\
\hline & Mean & 3.06 & 2.19 & 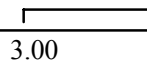 & $\overrightarrow{7}_{1.81}$ & \multirow[t]{2}{*}{12.79} \\
\hline \multirow[t]{2}{*}{ Most Likely to Purchase } & Median & 3 & 2 & 3 & 2 & \\
\hline & & 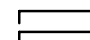 & $\neg$ & & & \multirow{3}{*}{7.00} \\
\hline \multirow{3}{*}{ Level of Trust } & Mean & 3.06 & 2.13 & 2.75 & 2.06 & \\
\hline & Median & 3 & 2 & 3 & 2 & \\
\hline & Mean & 2.00 & 2.88 & $\frac{\Gamma}{2.06}$ & ᄀ. 3.31 & \multirow{2}{*}{15.95} \\
\hline Level of Annoyance & Median & 2 & 3 & 2 & 3 & \\
\hline \multirow{2}{*}{ Level of Interference } & Mean & 2.38 & 2.56 & 2.25 & 2.44 & \multirow{2}{*}{ N.S } \\
\hline & Median & 2 & 2.5 & 2 & 2.5 & \\
\hline \multirow{2}{*}{ Level of Appropriateness } & Mean & 2.94 & 2.38 & 2.75 & 1.69 & \multirow{2}{*}{ N.S. } \\
\hline & Median & 3 & 2.5 & 2.5 & 1 & \\
\hline \multirow{2}{*}{ Level of Urgency } & Mean & 2.56 & 2.56 & 2.25 & 2.75 & \multirow{2}{*}{6.46} \\
\hline & Median & 2 & 3 & 2 & 3 & \\
\hline \multirow{2}{*}{ Overall Preference } & Mean & 2.81 & 2.06 & 2.94 & 2.19 & \multirow{2}{*}{5.28} \\
\hline & Median & 3 & 2 & 3 & 2 & \\
\hline
\end{tabular}

Indicates Significant Difference $(\mathrm{p}<0.05)$

\section{Discussion}

Two critical factors govern the benefit of notification systems in general and collision warnings in particular: the effect on performance and the effect on attitudes. Experiment 1 focused on driver performance and Experiment 2 focused on driver attitudes.

Experiment 1 showed that interface characteristics are a powerful influence on the performance of a warning system. Although, warning modality did not affect the number of collisions or the safety margin, the warning strategy had a substantial effect. Graded warnings led to a larger minimum time to collision, indicating a greater margin of safety, than did single-stage warnings. Compared to graded warnings, the singlestage warnings tended to induce a greater number of inappropriate braking responses.

The effect of the graded alerts parallel the effect of graded alerts seen with a roadway condition warning system (e.g., alerts drivers to the likelihood of skidding) [9]. In that study, drivers with the graded warning demonstrated better vehicle control. Interestingly the benefit of the graded alert was seen in the present study even though there was a high rate of nuisance alarms, which have been shown to undermine trust and reliance [3].

Experiment 2 showed that graded haptic warnings were received more positively than single-stage auditory warnings. On all measures of acceptance summarized in Table 2 the graded haptic warning is preferred to the single-stage auditory warning. In general, the haptic warnings were preferred to the auditory warnings, suggesting that haptic warnings for invehicle systems merit further investigation.

\section{CONCLUSION}

Graded warnings provided a greater safety margin and there was no evidence that the graded warning habituates drivers to collision warnings and leaves them less prepared to respond to single-stage collision situations. Similarly, the graded warning also induced fewer inappropriate responses to the nuisance alarms, suggesting the graded approach may provide an additional safety benefit because it may reduce rear-end collisions induced by sudden braking in response to false warnings. Drivers trusted the graded warning more and there was no indication of increased annoyance associated with the greater number of alerts produced by graded strategy. In combination, these results suggest the graded warning is a promising design alternative.

The warning modality had little effect on driver performance-drivers performed similarly with haptic and auditory warnings. However, haptic warnings were preferred to the auditory warnings on several dimensions including trust, overall benefit to driving, and annoyance. These findings suggest that haptic displays may be a viable alternative to auditory displays. 
This study fills an important gap in our understanding of how the display parameters of a specific type of notification system, a warning, influence performance and acceptance [18]. The results point to the importance of considering interface characteristics in addition to the algorithms that trigger the warnings. The results also strongly suggest that non-standard warning mode (e.g., haptic cues from a vibrating seat) and warning strategies (e.g., a graded warning) need to be considered to promote appropriate use and acceptance.

These conclusions need to be verified in more realistic driving situations. The drivers in our studies experienced the system for only an hour and drivers' attitudes and performance after months of exposure might be quite different.

\section{ACKNOWLEDGMENTS}

This research was sponsored by the National Highway Traffic Safety Administration, Contract DTNH22-95-D-07168 IQC \#2(8-07633) and we thank Mike Perel, August Burgett, Wassim Najm, Robert Miller, and David Smith for their comments.

\section{REFERENCES}

[1] Ashley, S. Driving the info highway Scientific American, 2001.

[2] Bliss, J. and Acton, S.A. Alarm mistrust in automobiles: how collision alarm reliability affects driving. Applied Ergonomics, 34. 499-509.

[3] Bliss, J.P. and Dunn, M.C. Behavioural implications of alarm mistrust as a function of task workload. Ergonomics, 43 (9). 1283-1300.

[4] Brown, I.D., Tickner, A.H. and Simmonds, D.C.V. Interference between concurrent tasks of driving and telephoning. Journal of Applied Psychology, 53 (5). 419-424.

[5] Brown, T.L., Lee, J.D. and McGehee, D.V. Human performance models and rear-end collision avoidance algorithms. Human Factors, 43 (3). 462-482.

[6] Burgett, A.L., Carter, A., Miller, R.J., Najm, W.G. and Smith, D.L. A collision warning algorithm for rear-end collisions, National Highway Traffic Safety Administration, Washington, DC, 1998 .

[7] Diederich, A. Intersensory facilitation of reaction-time: Evaluation of counter and diffusion coactivation models. Journal of Mathematical Psychology, 39 (2). 197-215.

[8] Gielen, S.C.A.M. and Schnidt, R.A. On the nature of intersensory facilitation of reaction time. Perception and Psychophysics, 34. 161-168.

[9] Gupta, N., Bisantz, A.M. and Singh, T. The effects of adverse condition warning system characteristics on driver performance: an investigation of alarm signal type and threshold level. Behaviour \& Information Technology, 21 (4). 235-248.

[10] Haigney, D. and Westerman, S.J. Mobile (cellular) phone use and driving: a critical review of research methodology. Ergonomics, 44 (2). 132-143.

[11] Janssen, W. and Nilsson, L. Behavioural effects of driver support. in Parkes, A.M. and Franzen, S. eds. Driving Future Vehicles, Taylor \& Francis, Washington, D.C., 1993, 147-155.

[12] Kiefer, R., LeBlanc, D., Palmer, M., Salinger, J., Deering, R. and Shulman, M. Development and validation of functional definitions and evaluation procedures for collision warning/avoidance systems, Crash Avoidance Metrics Partnership, Washington DC, 1999.

[13] Lee, J.D., Caven, B., Haake, S. and Brown, T.L. Speech-based interaction with in-vehicle computers: The effect of speechbased e-mail on drivers' attention to the road. Human Factors, 43 (4). 631-640.

[14] Lee, J.D., McGehee, D.V., Brown, T.L. and Reyes, M.L. Collision warning timing, driver distraction, and driver response to imminent rear end collisions in a high-fidelity driving simulator. Human Factors, 44 (2). 314-334.

[15] Lee, J.D., McGehee, D.V., Brown, T.L. and Reyes, M.L. Collision warning timing, driver distraction, and driver response to imminent rear-end collisions in a high-fidelity driving simulator. Human Factors, 44 (2). 314-334.

[16] Lee, J.D., McGehee, D.V., Brown, T.L. and Reyes, M.L. Driver Distraction, Warning Algorithm Parameters, and Driver Response to Imminent Rear-end Collisions in a High-Fidelity Driving Simulator, National Highway Traffic Safety Administration, Washington, DC, 2002.

[17] Lee, J.D. and See, K.A. Trust in technology: Designing for appropriate reliance. Human Factors.

[18] McCrickard, D.S., Chewar, C.M., Somervell, J.P. and Ndiwalana, A. A Model for Notification Systems Evaluation: Toward Assessing Usability for Multitasking Activity.

[19] McCrickard, D.S., Czerwinski, M. and Bartram, L. Introduction: design and evaluation of notification user interfaces. International Journal of Human-Computer Studies, 58 (5). 509514.

[20] Nickerson, R.S. Intersensory facilitation of reaction time: Energy summation or preparation enhancement? Psychological Review, 80. 489-509.

[21] Parasuraman, R., Hancock, P.A. and Olofinboba, O. Alarm effectiveness in driver-centred collision-warning systems. Ergonomics, 40 (3). 390-399.

[22] Peters, G.A. and Peters, B.J. The distracted driver. Journal of the Royal Society For the Promotion of Health, 121 (1). 23-28.

[23] Raby, M., McGehee, D.V., Lee, J.D. and Norse, G.E. Defining the interface for a snowplow lane tracking device using a systems-based approach. Proceedings of the Human Factors and Ergonomics Society, 3. 369-372.

[24] Sklar, A.E. and Sarter, N.B. Good vibrations: Tactile feedback in support of attention allocation and human-automation coordination in event-driven domains. Human Factors, 41 (4). 543552.

[25] Sorkin, R.D., Kantowitz, B.H. and Kantowitz, S.C. Likelihood alarm displays. Human Factors, 30. 445-459.

[26] Tijerina, L., Jackson, J.L., Pomerleau, D.A., Romano, R.A. and Peterson, A. Run-Off Road Collision Avoidance Using IVHS Countermeasures: Task 3 Report - Volume II, U.S. Department of Transportation, Washington, D.C., 1995.

[27] Tijerina, L., Johnston, S., Parmer, E., Pham, H.A., Winterbottom, M.D. and Barickman, F.S. Preliminary Studies in Haptic Displays for Rear-end Collision Avoidance System and Adaptive Cruise Control Applications, National Highway Transportation Safety Administration, Washington, DC, 2000.

[28] Todd, J.W. Reaction time to multiple stimuli. Archives of Psychology, 3. 1-65. 